GENÈSE D'UN CHAMP SCIENTIFIQUE : L'ORTHOPÉDIE COMME FABRIQUE D'UNE GYMNASTIQUE. DU CHAMP MÉDICAL, DE LA SPÉCIALITÉ ORTHOPÉDIQUE ET DES EXERCICES CORPORELS (1817-1847).

\title{
Grégory Quin
}

L'Harmattan | Sciences sociales et sport

$2009 / 1-N^{\circ} 2$

pages 171 à 199

ISSN 1967-7359

Article disponible en ligne à l'adresse:

http://www.cairn.info/revue-sciences-sociales-et-sport-2009-1-page-171.htm

Pour citer cet article :

Quin Grégory, « Genèse d'un champ scientifique : l'Orthopédie comme fabrique d'une gymnastique. Du champ médical, de la spécialité orthopédique et des exercices corporels (1817-1847). »,

Sciences sociales et sport, 2009/1 №2, p. 171-199. DOI : 10.3917/rsss.002.0171

Distribution électronique Cairn.info pour L'Harmattan.

(c) L'Harmattan. Tous droits réservés pour tous pays.

La reproduction ou représentation de cet article, notamment par photocopie, n'est autorisée que dans les limites des conditions générales d'utilisation du site ou, le cas échéant, des conditions générales de la licence souscrite par votre établissement. Toute autre reproduction ou représentation, en tout ou partie, sous quelque forme et de quelque manière que ce soit, est interdite sauf accord préalable et écrit de l'éditeur, en dehors des cas prévus par la législation en vigueur en France. II est précisé que son stockage dans une base de données est également interdit. 
Revue Sciences sociales et sport $n^{\circ} 2$ - Septembre 2009

\title{
Genèse d'un champ scientifique : l'Orthopédie comme fabrique d'une gymnastique. \\ Du champ médical, de la spécialité orthopédique et des exercices corporels (1817-1847).
}

\author{
Grégory Quin $^{*}$
}

\section{Introduction}

Notre analyse tente de circonscrire l'économie des discours gymniques médicaux et orthopédiques dans la première moitié du XIX ${ }^{\mathrm{e}}$ siècle, sur la base de deux hypothèses. D’une part, nous considérons les années entre 1817 et 1847 comme déterminantes dans l'institutionnalisation de l'orthopédie, par la multiplicité des acteurs engagés ; et, d'autre part, nous entendons les discours orthopédiques comme une matrice du procès de systématisation des pratiques d'exercice corporel dans la période post-révolutionnaire en France ${ }^{1}$. Nous chercherons notamment à poursuivre et enrichir les recherches initiées par Georges Vigarello dans Le corps redressé ; celui-ci déclarait vouloir scruter l'invention et le développement des exercices physiques à travers les pratiques de redressement des corps, mais encore il l'a fait sans réellement prendre en compte la dynamique institutionnelle propre à la médecine et plus précisément à la spécialité orthopédique, comme nous nous proposons de le faire.

Nous envisageons ainsi de construire une histoire critique de la gymnastique au cours de la première moitié du $\mathrm{XIX}^{\mathrm{e}}$ siècle, où le colonel Amoros n'occuperait pas tout seul le devant de la scène, pour nous plonger dans le cœur de la fabrique de la gymnastique. Si Amoros occupe une position dominante dans l'espace des pratiques d'exercice corporel, et si son influence sur la gymnastique française est irrécusable, y compris dans les discours gymniques médicaux, sa capacité d'innovation tout comme ses connaissances "scientifiques" semblent pour le moins discutables et nous verrons qu'elles sont discutées. Formé à l'école pestalozzienne à Madrid, Amoros fait : « [...] ses débuts [en France] dans une maison d'éducation - l'Institution des Nations européennes [...] -, il débute en juillet 1817 avec une quinzaine d'élèves, à faire

\footnotetext{
1 A propos de l'histoire de l'orthopédie et du redressement des corps, on lira VigARELlO, Georges. 2001. Le corps redressé. Histoire d'un pouvoir pédagogique, Paris, Armand Colin (tout particulièrement les chapitres IV et V à propos de la première moitié du XIX ${ }^{\mathrm{e}}$ siècle).
} 
Genèse d'un champ scientifique : l'Orthopédie comme fabrique d'une gymnastique.

l'application de son système d'éducation physique et morale. » ${ }^{2}$ Le colonel Amoros n'innove pas pratiquement, mais imprime une dimension nationale au mouvement des exercices corporels français ${ }^{3}$, notamment par l'enseignement qu'il développe dans les casernes militaires. Au-delà de son action dans l'éducation des soldats, le colonel Amoros revendique une certaine légitimité scientifique, qui lui sera notamment conféré par le médecin Casimir Broussais, dont on connaît l'importance dans une généalogie de l'intrication du pédagogique et du médical, notamment par sa participation à la Société de phrénologie ${ }^{4}$ dans les années 1830.

Les rapports du pédagogique et du médical - comme nombre de lieux sociaux - sont transformés dans les années 1789-1815, et la Révolution française, d'abord mutation politique, doit aussi être pensée comme une révolution scientifique et pédagogique. En effet, l'architecture de la médecine en France se trouve complètement transformée au lendemain de la Révolution française. Les anciennes facultés ont été démantelées, les corporations de médecins et de chirurgiens sont supprimées. Dès 1795, de nouvelles écoles de médecine sont créées, la séparation entre médecine et chirurgie est abolie, et un nouveau cadre juridique est défini quant à l'exercice de la médecine. L’hôpital va désormais jouer un triple rôle « de soins aux malades indigents, de terrain privilégié d'observation des maladies et de lieu de formation clinique des étudiants $»^{5}$. Les savoirs vont se trouver bouleversés, par l'introduction d'une nouvelle conception de la clinique. L'ancienne théorie humorale est abandonnée au profit d'une nouvelle approche qui cherche désormais à localiser la pathologie dans les tissus constituant le corps. Lésions tissulaires contre déséquilibres humoraux, c'est dans cette opposition proprement épistémologique que va venir se loger le moteur du processus de spécialisation. Encore entre «[...] 1770 et 1825, on voit se développer parallèlement deux catégories du discours médical : l'un est de caractère scientifique, l'autre de

\footnotetext{
${ }^{2}$ Амүот, С. J. B. 1852. Histoire du Colonel Amoros, de sa méthode d'éducation physique et morale, Paris, Colas.

${ }^{3}$ A ce propos, on lira Defrance, Jacques. 1987. L'excellence corporelle, Rennes, Presses Universitaires de Rennes.

${ }^{4}$ Sur l'histoire de la phrénologie ; RENNEVILLE, Marc. 2000. Le langage des crânes, Paris, Empêcheurs de penser en rond.

${ }^{5}$ Pinell, Patrice. 2005. "Champ médical et processus de spécialisation », Actes de la recherche en sciences sociales, tome $156-157, \mathrm{n}^{\circ} 1-2, \mathrm{p} .7-8$.
} 
Revue Sciences sociales et sport $n^{\circ} 2$ - Septembre 2009

caractère littéraire et moraliste $»^{6}$. Si c'est autour de cette double potentialité que vont se développer les discours médicaux au tournant de l'âge classique, le phénomène principal à souligner en introduction du présent travail est bien celui de la spécialisation que va connaître le champ médical dès l'aube du XIX ${ }^{\mathrm{e}}$ siècle et qui le structure jusqu'au $\mathrm{XX}^{\mathrm{e}}$ siècle. Ce processus de spécialisation va s'articuler avec la mise au point de thérapeutiques basées sur un appareillage et/ou un savoir-faire mobilisant des compétences techniques, comme en ophtalmologie ou en orthopédie. La généalogie de la spécialisation en médecine $^{7}$ révèle toutes les implications, à la fois intellectuelles, sociales, économiques, institutionnelles et idéologiques ${ }^{8}$ d'un tel processus. Nous suivrons notamment l'évolution de la légitimité des exercices du corps ${ }^{9}$, à travers leur utilisation à des fins hygiéniques et thérapeutiques - à côté de pratiques mécaniques ou chirurgicales.

Il s'agit de voir quelle forme prend l'institutionnalisation de l'investissement des corps (masculins et féminins) au sein de l'orthopédie, et comment les acteurs de la spécialisation contribuent à alimenter le processus de systématisation des pratiques d'exercice corporel, en nous appuyant notamment sur les controverses méthodologiques, pratiques et symboliques, inhérentes au processus de structuration de l'espace social médical, et particulièrement à la formation de l'orthopédie. Partie d'une épistémè moderne, les discours orthopédiques structurent un espace médical autour des affections et des difformités du corps humain. Ils ont une certaine légitimité, et semblent liés par un projet commun de redressement des corps déviés. Les discours, comme ensemble de significations contraintes et contraignantes, structurent les rapports sociaux. Enchâssés dans des systèmes de valeurs et de normes, les discours

\footnotetext{
${ }^{6}$ KNIBIELHER, Yvonne 1976. « Les médecins et la "nature féminine” au temps du code civil », Annales E.S.C., $31^{\text {ème }}$ année, n 4, p. 828.

${ }^{7}$ WeIsz, Georges. 2006. Divide and Conquer. A Comparative History of Medical Specialisation, Oxford, Oxford University Press.

${ }^{8}$ WEISZ, Georges. 1994. " Mapping medical specialization in Paris in the nineteenth and twentieth centuries ", Social History of Medicine, 7, p. 178, cité dans PINELL, Patrice. 2005. Art. cit., p. 6.

${ }^{9}$ Nous suivrons en cela les analyses de Jacques Defrance sur le développement et la légitimation des gymnastiques : DEFRANCE, Jacques. 1987. L'excellence corporelle, Rennes, Presses Universitaires de Rennes (notamment le chapitre 3 sur «La légitimation. La production de la demande : processus et stratégies de légitimation de la pratique d'exercice » et le chapitre 4 sur «La différenciation. Les usages sociaux de l'exercice : différenciation des pratiques et des principes de légitimation »).
} 
Genèse d'un champ scientifique : l'Orthopédie comme fabrique d'une gymnastique.

recomposent les normes ; et ce caractère à la fois « dépendant et producteur des normes » détermine la performativité des discours, au sein d’une épistémè. Pierre Bourdieu, affirmant sa volonté de dépasser cette posture épistémologique totalisante, précise qu'il : «[...] n'est pas possible, même dans le cas du champ scientifique, de traiter de l'ordre culturel (l'épistémè) comme totalement indépendant des agents et des institutions qui l'actualisent et le portent à l'existence, et d'ignorer les connexions socio-logiques qui accompagnent et sous-tendent les consécutions logiques [...]. " ${ }^{10}$ De fait, les événements biographiques - privés et scientifiques - des différents acteurs pourront alors esquisser les contours d'une fabrique de la gymnastique dans les années 18201850, en soulignant les controverses inhérentes à l'institutionnalisation de l’orthopédie.

Une telle lecture du processus de systématisation des pratiques d'exercice corporel, dans la première moitié du XIX ${ }^{\mathrm{e}}$ siècle, donne aux discours gymniques médicaux une vraie dimension explicative, à la fois dans l'ordre du discours médical et dans l'ordre du discours gymnastique. Ainsi, l'on peut dire que, comme toute production symbolique, les exercices corporels ne peuvent être intelligibles hors d'une posture les inscrivant à la fois dans les structures sociales et dans les imaginaires sociaux, considérant ces exercices comme des formes symboliques ${ }^{11}$. Mais encore, il nous semble impossible d'accéder à une intelligibilité satisfaisante pour la structuration et le développement d'un espace social, en dehors du "recours aux notions de stratégies et de trajectoires qui restituent la marge de manœuvre des acteurs et permettent d'en reconstruire le sens $»^{12}$. Dans cette mesure, les discours recueillis par l'enquête historique révèlent les projets, les innovations et les controverses inhérentes à un processus de spécialisation comme celui de l'orthopédie. Entre un discours sur la dégénération dans la seconde moitié du $\mathrm{XVIII}^{\mathrm{e}}$ siècle et les développements d'une pensée de la dégénérescence après les travaux du docteur Morel ${ }^{13}$, on

\footnotetext{
${ }^{10}$ BourdiEU, Pierre. 1992. Les règles de l'art. Genèse et structure du champ littéraire, Paris, Seuil, p. 326-327 [souligné par moi].

${ }^{11}$ Bourdieu, Pierre. 1977. " Sur le pouvoir symbolique », Annales. E.S.C., $32^{\text {ème }}$ année, $\mathrm{n}^{\circ}$ 3, p. 405-411.

12 BENSA, Alban. 1996. «De la micro-histoire, vers une anthropologie critique », in REVEL, Jacques (Dir.). Jeux d'échelles. La micro-analyse à l'expérience, Paris, Gallimard-Seuil, p. 46 [souligné par moi].

13 Morel, Benedict-Augustin. 1857. Traité des dégénérescences physiques, intellectuelles et morales de l'espèce humaine, Paris, Baillière.
} 
Revue Sciences sociales et sport $n^{\circ} 2$ - Septembre 2009

observe le déploiement d'un réel moment orthopédique durant les années de la Restauration et de la Monarchie de Juillet.

Les sources mobilisées pour circonscrire l'économie des discours gymniques médicaux et orthopédiques sont très diverses : traités orthopédiques, articles de revues, prospectus d'établissements orthopédiques, articles de dictionnaires, rapports académiques, concours académiques, etc. La diversité des sources illustre aussi la polysémie des processus de spécialisation de l'orthopédie et de systématisation des pratiques d'exercice corporel, entre 1817 et la fondation de la gymnastique française par le Colonel Amoros, et 1847 date d'entrée de la gymnastique à l'hôpital. Tous ces lieux de la mémoire médicale illustrent la complexité du processus et justifient l'utilité d'une histoire à différentes échelles qui observe les différents acteurs, leur inscription dans des parcours de vie singuliers, leurs controverses et leurs capacités d'innovation individuelle et collective, sans omettre absolument le poids crucial du contexte, marqué par l'affirmation d'une biopolitique et d'un habitus social bourgeois.

Nous recourrons aux concepts de " champ ${ }^{14}$ et de «biopolitique " à des fins heuristiques, pour tenter d'articuler les différents points de vue, les replacer les uns par rapport aux autres, ainsi que pour dévoiler l'historicité de la production des gymnastiques dans la première moitié du XIX ${ }^{\mathrm{e}}$ siècle.

\section{I - L'orthopédie. Structuration d'un champ scientifique}

Après la suppression des corporations de médecins et de chirurgiens pendant la phase de radicalisation de la Révolution française (1792-1794), le Consulat va accélérer la reconstruction de l'espace social médical entamé après la chute de Robespierre. La loi du 10 mars 1803, instaure des écoles de médecine, abolit la distinction entre médecins et chirurgiens, et institue deux niveaux à la pratique médicale : celui des docteurs, issus des écoles de médecine devenues facultés en 1808, et dont le titre conférait le droit d'exercer la médecine et la chirurgie sur l'ensemble territoire ; celui des officiers de santé, pratiquant une médecine restreinte, après des études plus brèves, et limitée au département d'obtention de l'officiat. Si deux niveaux d'exercice de la médecine continuent d'exister jusqu'en 1892, ce sont désormais les médecins qui tiennent le haut du pavé dans le champ médical, soutenus par un cadre

\footnotetext{
14 Sur le concept de «champ », on se réfèrera aux travaux de Pierre Bourdieu : BouRdiEU, Pierre. 1971a. " Genèse et structure du champ religieux ", Revue française de sociologie, tome 12, $\mathrm{n}^{\circ}$ 3, p. 295-324 ; BOURDIEU, Pierre. 1971b. "Une interprétation de la théorie de la religion selon Max Weber ", Archives européennes de Sociologie, tome XII, p. 3-21.
} 
Genèse d'un champ scientifique : l'Orthopédie comme fabrique d'une gymnastique.

législatif en leur faveur et qui tend à minimiser le rôle d'autres acteurs de la popularisation des pratiques médicales. Malgré tout - et particulièrement en orthopédie - une pluralité d'acteurs continue à travailler. Ainsi, la catégorie de « médecin » ${ }^{15}$ vaut aussi pour ceux qu'elle n'inclurait pas parmi les acteurs de la spécialisation orthopédique : les mécaniciens, les gymnasiarques, les «fils de médecin » ou les autodidactes. En effet, la constitution d'un espace médical spécialisé orthopédique n’est pas un processus exclusivement dirigé par des docteurs en médecine qui construiraient ainsi leur propre corpus de savoirs à des fins de légitimation interne à leur spécialité et au champ médical. Nous nous proposons d'aborder la question de la spécialité orthopédique à partir des acteurs et de leur engagement en faveur des exercices corporels. A des fins heuristiques, nous essayerons de présenter ces acteurs engagés dans la construction de spécialité orthopédique en trois groupes.

Dans un premier temps, nous verrons les quelques médecins les plus actifs dans la nouvelle spécialité orthopédique, dès la première moitié du XIX ${ }^{\mathrm{e}}$ siècle. Ces principaux médecins sont Jules Guérin, Sauveur-Henri-Victor Bouvier, Vincent Duval et Charles-Gabriel Pravaz dont les travaux dessinent, entre 1820 et 1840, l'architecture de la spécialité orthopédique pour un long $\mathrm{XIX}^{\mathrm{e}}$ siècle. Ces quatre premiers noms sont retenus comme " principaux » en raison de la multiplicité de réseaux, institutions ou encore revues auxquels ils participent. Ils intègrent les Académies, ils dirigent des revues - ils y publient des comptes-rendus et des articles -, ils rédigent des notices dans les grands dictionnaires médicaux, leurs travaux sont cités par d'autres agents du champ médical et ils sont très présents dans l'institution hospitalière dès les premières années d'introduction de l'orthopédie, puis de la gymnastique.

Quatre lettres de la main de Jules Guérin, consultées aux Archives des hôpitaux de Paris, témoignent des collaborations existant entre ces médecins. Ces lettres révèlent aussi que Jules Guérin et Charles Pravaz furent initialement co-directeurs de l'Institut orthopédique de Paris, qu'une collaboration a lié Jules Guérin et Vincent Duval, enfin que les orthopédistes cherchent à entrer - dès le milieu des années 1830 - dans les réseaux hospitaliers, pour «prendre des mesures comparatives et faire quelques observations sur la conformation des enfants ${ }^{16}$, et développer davantage leurs savoirs.

\footnotetext{
${ }^{15}$ Diplômé d'un doctorat en médecine obtenu dans une Faculté de médecine

${ }^{16}$ Lettre de Jules Guérin du 11 février 1836, Lettres manuscrites de médecins et chirurgiens. XIX ${ }^{\mathrm{e}}$ siècle. Archives de l'Assistance Publique - Hôpitaux de Paris (APHP), 706 FOSS 1.
} 
Revue Sciences sociales et sport $n^{\circ} 2$ - Septembre 2009

S'ils se sont vraisemblablement fréquentés en dehors même des lieux de la médecine stricto sensu, c'est encore au titre de leur position de directeurs d'établissements orthopédiques et gymnastiques dans les années 1820-1840 qu'ils forment un groupe homogène. Ces dispositifs de savoir/pouvoir leur donnent une possibilité exceptionnelle d'être en contact prolongé avec des pensionnaires souffrant de diverses difformités orthopédiques, et donc à la fois de comprendre et de guérir - notamment par des exercices physiques - ces affections du corps.

Le docteur Jacques-Mathieu Delpech n’est pas de la même génération, puisqu'il disparaît tragiquement à l'âge de 60 ans en 1832, et s'il ne participe pas directement de la vie médicale parisienne, l'importance qu'il va donner aux traitements gymnastiques - soulignée par les autres médecins orthopédistes parisiens - fait de lui un homme incontournable dans la dynamique sociale circonscrite, et nous oblige à l'intégrer à ce groupe des principaux orthopédistes du temps. De plus, il dirige pendant très longtemps un établissement orthopédique et gymnastique à Montpellier, ce qui lui donne un profil tout à fait semblable aux précédents orthopédistes parisiens.

A côté de ces orthopédistes, il existe un groupe tout à fait singulier et dont l'engagement dans la spécialité orthopédique la légitime dans son ensemble ; il s'agit de " grands noms » du champ médical, notamment autour de la Faculté de médecine de Paris. En effet, plusieurs «grands noms » du champ médical sont directement impliqués dans le processus de spécialisation que connaît l'orthopédie, nous pouvons citer Casimir Broussais, Charles Londe, Claude Lachaise, le baron Dupuytren, ou Louis-Jacques Bégin. Ils sont membres de l'Académie de médecine ${ }^{17}$, reconnus par leurs pairs, fils de médecins célèbres, et prennent part aux riches débats de leur temps. S'ils n'accordent pas tout leur temps à la constitution de savoirs et à la recherche de thérapeutiques orthopédiques ou gymnastiques, ils jouent au moins un rôle de légitimation auprès d'autres acteurs. Ils apportent alors à la fois leur capital spécifique, mais surtout un capital symbolique, inhérent à leur position dans le champ médical, et qu'ils peuvent faire valoir sur le marché ${ }^{18}$ des thérapeutiques

\footnotetext{
${ }^{17}$ L’Académie royale de médecine est créée en 1820 par Louis XVIII, avec l'appui du baron Antoine Portal.

18 Sur l'importance des connexions entre l'émergence d'un marché médical et l'institutionnalisation d'une nouvelle spécialité médicale, on lira MALPAS, Constance. 2004. « Jules Guerin Makes his Market : the Social Economy of Orthopaedic Medicine in Paris, c. 1825-1845 », in BleCOURT (de), Willem ; UsBorne, Cornelie (Dir.).
} 
Genèse d'un champ scientifique : l'Orthopédie comme fabrique d'une gymnastique.

pour le redressement des corps. Ce sont moins des praticiens et ils insistent davantage sur la théorisation des pratiques d'exercice corporel.

En ce qui concerne les démarches de légitimation d'établissements orthopédico-gymnastiques, nous avons, d'une part, Casimir Broussais ${ }^{19}$ qui occupe une position de "professeur de physiologie ${ }^{20}$ auprès du colonel Amoros lorsque ce dernier cherche à élargir son domaine d'action gymnastique, en associant le mot orthopédique dans le titre - et dans les pratiques - de son gymnase de Grenelle. Et, d'autre part, Guillaume Dupuytren ${ }^{21}$ qui patronne les tentatives de Madame Masson de la Malmaison pour la diffusion de l'orthopédie et de la gymnastique, notamment au sein des maisons d'éducation féminine de la Légion d'honneur, dès le début des années 1830.

Mais encore ces médecins ne doivent-ils pas uniquement être considérés comme des forces de légitimation. Ainsi, Claude Lachaise et Charles Londe vont aussi créer du savoir dans le domaine des exercices corporels et des thérapeutiques orthopédiques. Le docteur Londe publie un traité reconnu par ses pairs - La gymnastique médicale, en 1821 - dans lequel, il fixe les contenus des trois grandes catégories d'exercice du corps : actifs, passifs et mixtes. De son côté, le docteur Lachaise se lance dans une vive critique des méthodes de redressement appareillées, en particulier les lits mécaniques à extension très en vogue encore au début des années 1820. Par son activité auprès du colonel Amoros, Casimir Broussais s'attache aussi à penser les exercices du corps, et pour cela, il rédige en 1827 un ouvrage sur la gymnastique considérée comme un moyen hygiénique et comme agent thérapeutique, où il déclare que :

« La gymnastique n'est point seulement l'art d'exercer le corps, elle a encore pour but d'influer sur le moral en agissant sur le physique. C'est ce qu'a bien senti son fondateur [Amoros] en France, quand il l'a définie : La science raisonnée de tous nos mouvements, de leurs rapports avec nos sens, notre

Cultural Approaches to the History of Medicine. Mediating Medicine in Early Modern and Moderne Europe, New York, Palgrave Macmillan, p. 148-170.

${ }^{19}$ Casimir Broussais est le fils du célèbre François Broussais, l’une des figures tutélaires de la médecine française du premier tiers du $\mathrm{XIX}^{\mathrm{e}}$ siècle.

${ }^{20}$ Amoros, Francisco. 1827. Précis physiologique sur les courbures de la colonne vertébrale, ou Exposé des moyens de prévenir et corriger les difformités de la taille, particulièrement chez les jeunes filles, sans le secours des lits à extension, Paris, Delaunay.

${ }^{21}$ Chirurgien célèbre des premières années du $\mathrm{XIX}^{\mathrm{e}}$ siècle, il est fait baron par Napoléon, et travaille notamment sur les opérations chirurgicales de la colonne vertébrale : DuPUYTREN, Guillaume. 1812. Lithotomie, Thèse de concours pour la chaire de médecine opératoire, Paris. 
Revue Sciences sociales et sport $n^{\circ} 2$ - Septembre 2009

intelligence, nos sentiments, nos mœurs et le développement des nos facultés. La gymnastique fait partie de l'éducation, de la politique, de l'hygiène et de la thérapeutique, elle appelle donc les médiations de l'homme d'état et de l'instituteur, du philosophe et du médecin. » ${ }^{22}$

Ainsi, la gymnastique est pleinement pensée comme partie d'un dispositif biopolitique, susceptible de contrarier le développement de la dégénération de l'espèce humaine ${ }^{23}$, mais encore manque-t-il à Broussais une réelle expérience clinique des corps difformes ${ }^{24}$. Autre partisan de la gymnastique d'Amoros, dès les années 1820, Louis-Jacques Bégin développe une analyse sensiblement identique à celle de Broussais : dans son article « orthopédie » dans le Dictionnaire des Sciences Médicales - le Panckoucke - il souligne :

« [...] l'imperfection de notre système d'éducation, qui, loin d'être propre à perfectionner à la fois le physique et le moral, néglige complètement le premier, et fait ainsi acquérir à un grand nombre de sujets cette débilité que l'on a mal à propos considérée comme favorable au développement de l'esprit. Nous savons que l'exercice continuel d'un organe en accroît la force, mais, avant d'agir, le cerveau a besoin de percevoir, comme les sens d'observer, et par conséquent les facultés physiques doivent jouir de toute leur énergie [...]. C’est donc, il faut le répéter sans cesse, [...] par une éducation convenablement dirigée que l'on épuisera enfin la source de ces tempéraments scrofuleux, rachitiques, etc., qui font depuis si longtemps le désespoir des médecins, et que l'on considère avec raison comme les causes les plus répandues et les plus puissantes de la dégénération de l'espèce humaine. L'ensemble des moyens les plus propres à inspirer aux enfants des sentiments nobles et généreux, à donner à leur corps une force et une agilité considérables, à orner leur esprit de connaissances étendues, doit fixer, actuellement surtout que l'on s'occupe de

22 BRoussais, Casimir. 1827. De la Gymnastique, considérée comme moyen thérapeutique et hygiénique, Paris, Lachevardière fils, p. 4.

${ }^{23}$ Le souci de contrarier la dégénération de l'espèce humaine émerge dans la seconde moitié du XIX ${ }^{\mathrm{e}}$ siècle, et il a été particulièrement bien scruté par Michel Foucault. Voir FouCAUlt, Michel. 2004. Sécurité, territoire, population. Cours au Collège de France. 1977-78, Paris, Gallimard-Seuil.

${ }^{24}$ Celle-là même qui permettra à Jules Guérin d'établir sur une base véritablement physiologique la régénération, en montrant que celle-ci existe au niveau musculaire et qu'elle est liée au temps et au degré de l’exercice « fonctionnel » qui lui est appliqué. A ce propos, on lira PociEllo, Christian. 1999. La Science en mouvement, Paris, PUF, p. 57 et suiv. 
Genèse d'un champ scientifique : l'Orthopédie comme fabrique d'une gymnastique.

toute part du perfectionnement de nos institutions, l'attention de tout ceux qui font du bonheur de l'homme, l'objet de leurs méditations. Nous l'avons déjà dit par ailleurs, le problème de l'éducation, dans un état libre, se rattache à tout ce qu'il y a de plus important et de plus élevé dans le système social : c'est le lien qui unit les sciences morales et politiques à la médecine ; c’est la clé de tout ce que l'on peut établir de salutaire, soit au moral, soit au physique. ${ }^{25}$

Le développement des savoirs et des pouvoirs de l'orthopédie implique donc des acteurs du champ médical au-delà de la stricte pratique médicale orthopédique. La question du redressement des corps prend une réelle dimension hygiénique et pédagogique, sous la plume des « grands noms » de la médecine. Extérieurs à la nécessité de «constituer » les savoirs proprement orthopédiques, ils peuvent discourir de manière plus générale (ou philosophique), et enrichir ainsi l'ensemble du corpus. Au fur et à mesure de la construction des savoirs de l'orthopédie et de la gymnastique, ces grands médecins vont en outre de plus en plus collaborer avec les acteurs du premier groupe, qui accèdent - comme nous l'avons vu - dans les années 1830-1840 à de réelles positions de pouvoir. Ainsi, les docteurs Bégin, Londe et Bouvier collaborent-ils pour l'évaluation - commanditée par l'Académie de médecine de la méthode de gymnastique de Clias ${ }^{26}$.

La volonté d'éclaircir les processus sociaux oblige l'historien a faire des choix, aussi le choix de proposer trois groupes amène à présenter un troisième groupe plus éclaté, dont les membres occupent un vaste spectre de positions dans la dynamique orthopédique. Ces acteurs, que l'on qualifiera de "secondaires", ne doivent pas être minimisés par cette appellation. Ils participent pleinement à l'élaboration des connaissances de l'orthopédie, quand bien même ils ne sont pas médecins et malgré l’opposition systématique que

25 Fournier-Pescay, François ; Begin, Louis-Jacques. 1819. " Orthopédie », Dictionnaire des sciences médicales, Paris, Panckouke, tome 38, p. 299-301.

${ }^{26}$ BEgin, Louis-Jacques ; LONDE, Charles ; BouviER, Saveur-Henri-Victor. 1845. « Sur la gymnastique populaire de M. Clias », Bulletin de l'académie de médecine, tome XI, Paris, Baillière. Le rapport a été fait à l’Académie royale de médecine le 21 octobre 1845. Le gymnasiarque Peter Heinrich Clias débutera sa carrière en Suisse, notamment à Berne, avant de la poursuivre en Angleterre puis en France. Il ne développe pas de système d'exercices physiques national, ni en Suisse, ni en France ; néanmoins ses travaux comptent parmi les premières tentatives de systématisation des pratiques d'exercice corporel. 
manifestent parfois les principaux médecins à leur encontre ${ }^{27}$. Ainsi, les docteurs Maisonabe, Leuchsenring-Hermann, Bienaimé, ou Laguerre, les bandagistes Hossard ou Duvoir, le gymnasiarque Amoros ou la directrice de l'établissement orthopédique et gymnastique de la maison d'éducation de la Légion d'Honneur Madame Masson de la Malmaison contribuent pleinement aux débats méthodologiques. Ils développent souvent des stratégies thérapeutiques et/ou hygiéniques - empruntées à d'autres acteurs -, dans leurs écrits, dans leurs ateliers de fabrication de corsets ou dans leurs directions d'établissements orthopédiques, gymnastiques ou même parfois hydrothérapiques. En 1833, l'Almanach du commerce de Paris ${ }^{28}$ recense 43 herniaires et bandagistes et 74 fabricants de corsets, ce qui atteste aussi de l'acuité avec laquelle se pose la question du rétablissement des déviations corporelles dans la capitale. Installés à leur compte, les herniaires et les bandagistes sont les petits artisans du redressement des corps. Unis par une logique plus d'une fois imparfaite, les discours produits par ces acteurs sont souvent porteurs d'un message ou de thérapeutiques anciennes, et parfois éculées. Et s'ils sont moins les vecteurs d'une innovation orthopédique, leur nombre et leur répartition à Paris attestent de l'existence d'un marché du redressement orthopédique des corps. Ils constituent la trame de l'orthopédie, et ils sont sans doute également des vecteurs préférentiels d'une diffusion de savoirs. Sollicité par un médecin ou un chirurgien « [...] le mécanicien, lorsque son secours est jugé nécessaire, ne doit s'occuper que de la construction des machines qui atteignent le mieux le but assigné par le chirurgien. ${ }^{29}$

Mais probablement aussi sollicités directement par des familles, dans l'impossibilité de faire admettre leurs enfants atteints de difformités dans les établissements orthopédiques ${ }^{30}$, les fabricants de corsets ou les mécaniciens bénéficient aussi d'une très grande autonomie dans l'application de thérapeutiques, et cela au moins jusqu’à la fin de la première moitié du XIX

\footnotetext{
27 De nombreuses controverses médicales - théoriques, tout autant que pratiques, et même symboliques - émaillent l'histoire de l’orthopédie dans la première moitié du $\mathrm{XIX}^{\mathrm{e}}$ siècle. Nous le verrons dans la seconde partie à partir de deux exemples précis.

${ }^{28}$ BotTin, Sébastien. 1833. Almanach du commerce de Paris, des départements de l'Empire français et des principales villes de l'Europe, $X X X V I^{\text {ème }}$ année de publication, Paris, Bureau de l’Almanach.

${ }^{29}$ Fournier-PesCAY, François ; Begin, Louis-Jacques. 1819. Op. cit., p. 297.

${ }^{30}$ Le coût d'une année dans un établissement orthopédique et gymnastique à Paris dépasse sans doute fréquemment 2000 francs par pensionnaire, ce qui ne permet qu’à une toute petite partie de la bourgeoisie et de la noblesse de Paris de bénéficier des bienfaits d’un redressement contrôlé par un médecin au quotidien.
} 
Genèse d'un champ scientifique : l'Orthopédie comme fabrique d'une gymnastique.

siècle. Plusieurs explications à ce phénomène peuvent être avancées, d'une part, les principaux orthopédistes - Bouvier, Guérin, Duval - ne sont pas toujours en faveur des mêmes thérapeutiques et continuent à expérimenter différentes méthodologies, d'autre part, la structure du marché du redressement des corps reste encore relativement lâche ${ }^{31}$, avant l'institutionnalisation hospitalière définitive dans les années 1840, et enfin, les vertus des exercices corporels, si elles sont avérées et reconnues, peinent encore à se diffuser. De fait, les anciennes méthodes - plus rudes, voire violentes - de redressement peuvent ainsi perdurer.

Dans ce qui ressemble à des champs en cours de structuration - celui des pratiques d'exercice corporel, celui de la médecine -, nous souhaiterions souligner combien les questions de stratégies et de trajectoires sont décisives pour une lecture de l'investissement médical dans l'éducation des corps, à partir de la démarche médicale d'investigation méthodique de ces mêmes corps ${ }^{32}$. Davantage que les gymnasiarques, les médecins sont porteurs d'un discours systématique, légitimé par une institution. Ainsi, nous pourrions aller jusqu'à subsumer nos trois groupes sous les trois appellations de "prophètes", de "prêtres » et de «sorciers ", où les médecins-orthopédistes seraient les prophètes d'une nouvelle orthopédie, les "grands noms » seraient les prêtres d'une médecine révolutionnée et où les acteurs secondaires seraient les sorciers, dépourvus de la légitimité institutionnelle, mais devant faire face à des situations ordinaires, et gérant en somme le quotidien des déviations corporelles.

Paradoxe de l'histoire, c'est un acteur issu de ce dernier groupe - un « sorcier » donc -, Napoléon Laisné, qui prend la direction de la gymnastique dans l'institution hospitalière après 1847. Il rencontre d'ailleurs un franc succès notamment dans le traitement de la chorée - ou danse de Saint-Guy - à l’Hôpital des Enfants Malades. Par ses écrits ${ }^{33}$ et leur reconnaissance au sein

\footnotetext{
31 Avant l'entrée des traitements orthopédiques à l'hôpital durant les années 1830, aucun acteur, ni aucun lieu ne possède suffisamment de légitimité pour s'imposer et distordre - à son avantage - l'architecture du marché du redressement du corps.

32 Bourdieu, Pierre. 1971a. Art. cit. ; Bourdieu, Pierre. 1971b. Art. cit.

33 LAISNE, Napoléon. 1850. Gymnastique pratique, contenant la description des exercices, la construction et le prix de machines, et des chants spéciaux inédits : ouvrage destiné aux familles, aux établissements d'éducation, aux corps militaires, Paris, Dumaine ; LAISNE, Napoléon. 1865. Applications de la gymnastique à la guérison de quelques maladies, avec des observations sur l'enseignement actuel de la
} 
Revue Sciences sociales et sport $n^{\circ} 2$ - Septembre 2009

même du champ médical, Laisné contribue à la poursuite des réflexions autour d'une gymnastique médicale sous le Second Empire et les débuts de la Troisième république.

\section{II - Médecins contre praticiens ? Les controverses méthodologiques, pratiques et symboliques}

Nous souhaiterions prolonger l'analyse, à partir de controverses centrées autour de la question orthopédique dans les années 1820 à 1840. Parmi les nombreuses pathologies relevant de l'orthopédie, nous nous focaliserons spécifiquement sur les déviations ou difformités de la colonne vertébrale, laissant de côté les pieds-bots ou les difformités des membres supérieurs ou inférieurs, suivant en cela, la concentration discursive des orthopédistes euxmêmes $^{34}$. Il ne faudrait pas faire une lecture trop mécaniste de l'histoire qui est envisagée. Si les discours des médecins, des académies, des revues et des petites institutions semblent travailler de concert à la structuration de la spécialité orthopédique et à la systématisation de pratiques d'exercice corporel, ce sont bien les oppositions et les controverses dans le champ médical, tout à la fois méthodologiques, pratiques et symboliques, qui donnent du corps au processus.

La première de ces controverses oppose le colonel Amoros et le docteur Claude Lachaise autour de l'année 1827, sur la question de la «compétence médicale » du colonel Amoros dans l'emploi des méthodes thérapeutiques orthopédiques appareillées. Le docteur Lachaise refuse cette compétence au colonel Amoros pour deux raisons, en grande partie sans doute parce que le colonel Amoros n'est pas médecin - donc illégitime à produire des discours gymniques médicaux - ; et en raison de son opposition personnelle aux redressements de la colonne vertébrale à l'aide de machines orthopédiques ${ }^{35}$ comme les pratique Amoros dans son Gymnase de Grenelle. Cette position est

gymnastique, Paris, Leclerc ; LAISNE, Napoléon. 1870. Observations sur l'enseignement actuel de la gymnastique civil et militaire, Paris, Hachette.

${ }^{34}$ Les difformités de la colonne vertébrale sont l'objet d'une grande attention de la part des orthopédistes, qui lui consacrent une grande part de leurs travaux et de leurs traitements au sein de leurs établissements orthopédiques.

${ }^{35}$ LACHAISE, Claude. 1827. Précis physiologiques sur les courbures de la colonne vertébrale, ou exposé des moyens de prévenir et de corriger les difformités de la taille, particulièrement chez les jeunes filles, sans le secours des lits mécaniques à extension, Paris, Villeret ; LACHAISE, Claude. 1828. Nouvelles preuves des dangers des lits mécaniques et des avantages des exercices de gymnastiques dans le traitement des difformités de la taille, Paris, Villeret. 
Genèse d'un champ scientifique : l'Orthopédie comme fabrique d'une gymnastique.

d'ailleurs relayée à la même période par d'autres médecins dont Charles Londe, ainsi que Louis-Jacques Bégin ${ }^{36}$. Si le colonel Amoros se défend en insistant sur la rareté des visites du docteur Lachaise dans son établissement - prétextant le caractère infondé de ses critiques - et en s'appuyant sur la légitimité accordée à ses connaissances physiologiques par plusieurs médecins célèbres ${ }^{37}$, d'autres témoignages viennent corroborer les dires du docteur Lachaise, et permettent de douter sincèrement des capacités "médicales " amorosiennes. Le principal ne se joue pas dans la capacité à juger les travaux et compétences des uns et des autres, mais il faut souligner l'acuité avec laquelle se pose la question de la compétence médicale, autour du cœur de la gymnastique de l'époque - c'est-àdire autour du Gymnase Normal du colonel Amoros. Si l'on trouve des médecins dans les deux parties, c'est bien autour de la compétence médicale discutée - du colonel Amoros que se passe le débat. Au-delà, ce sont les différentes méthodes de redressement des colonnes vertébrales qui opposent les médecins engagés dans la controverse, et en particulier la méthode gymnastique, dont le statut hygiénique s'affirme, mais dont les potentialités curatives restent à démontrer. La force et, d'une certaine manière, la violence que l'on va introduire dans le redressement de la colonne vertébrale par l'intermédiaire des lits mécaniques ne sont pas du goût de tous les médecins. Il n'est pas fortuit non plus que cela soit le représentant d'une gymnastique à la française qui soit l'objet de ces critiques de la part du corps médical. En effet, celui-ci constate que le colonel Amoros se forge rapidement une certaine tutelle sur les exercices du corps ; et c'est bien contre le " maître " de la gymnastique française dans les années 1820 et 1830, que va se dresser Claude Lachaise, cherchant à imposer une vision davantage médicalisée du mouvement rationnel des corps sains ou malsains.

Ligne de faille de l'espace social médical d'un long $\mathrm{XIX}^{\mathrm{e}}$ siècle $^{38}$, l'opposition entre médecins et officiers de santé ne semble pas se poser avec une grande acuité pour l'orthopédie ${ }^{39}$; la remise en question de la capacité à

\footnotetext{
${ }^{36}$ Louis-Jacques Bégin occupe une position très singulière et ambiguë, dans la mesure où il se veut à la fois, soutien des exercices du colonel Amoros, directeur d'une classe de gymnastique dans le gymnase d'Amoros, et opposant aux méthodes de redressement mécaniques.

${ }^{37}$ Parmi lesquels, il faut compter : Broussais (fils), Esquirol, Portal, Roux, Virey, etc., tous déjà ou futurs membres de l'Académie de médecine de Paris.

${ }^{38}$ De 1803 à 1892 (date de suppression officielle de l'officiat de santé).

${ }^{39}$ Nous savons pourtant que des officiers de santé ont aussi pratiqué l'orthopédie, et notamment la chirurgie orthopédique - avec plus ou moins de succès - comme en
} 
Revue Sciences sociales et sport $n^{\circ} 2$ - Septembre 2009

soigner - concentrée ailleurs autour de la figure de l'officier de santé - se déplace vers les gymnasiarques et les mécaniciens ou bandagistes, ainsi que nous allons le voir dans la seconde controverse.

Celle-ci oppose Jules Guérin et Jules-Louis Hossard, mais associe également Sauveur-Henri-Victor Bouvier, ainsi que quelques autres orthopédistes et/ou médecins, autour de 1835, et s'articule autour de l'affirmation des traitements chirurgicaux des déviations de la colonne vertébrale - traitements pour lesquels Jules Guérin prendra fait et cause. La source principale mobilisée pour rendre compte de ce débat est la Gazette médicale de Paris $^{40}$, et notamment les comptes rendus des séances de l'Académie. Dans les années 1830, ce journal possède un large rayonnement, dans la mesure où il s'avère être la voix du doyen de la Faculté de médecine de Paris, Mathieu Orfila, grand ordonnateur de la médecine sous la Monarchie de Juillet, et proche du roi des Français, Louis-Philippe. Au delà des articles de la Gazette, l'intensité du questionnement orthopédique et gymnastique se traduit par la parution de traités (dans le sillage du traité de Charles Londe paru en 1821) et par la fondation d'établissements orthopédiques et gymnastiques dans Paris surtout, mais aussi ailleurs en France ${ }^{41}$. Autour de Jules Guérin et de Sauveur Bouvier, nous repérons une lutte pour la constitution d'un savoir orthopédique à travers le questionnement des méthodes thérapeutiques orthopédiques, au sein de laquelle, la gymnastique joue un rôle de trait d'union entre les différents agents du champ médical. Si Guérin ne jure presque plus que par la ténotomie ${ }^{42}$ - ou myotomie rachidienne -, diminuant en cela l'importance de la gymnastique dans le traitement des déviations de la colonne vertébrale, d'autres médecins - dont le docteur Bouvier - ou « soignants » témoignent de l'efficacité de leurs méthodes de redressement, s'appuyant en partie sur les

témoigne le passage dans Madame Bovary (1856), par Gustave Flaubert, où l'on voit Charles Bovary - officier de santé - saccager le pied bot du garçon d'écurie avant de finalement faire appel au médecin. Voir COOTER, Roger. 2004. "Orthopédie ", in LECOURT, Dominique (Dir.). Dictionnaire de la pensée médicale, Paris, PUF, p. 822827.

${ }^{40}$ La Gazette médicale de Paris est un journal médical hebdomadaire, dirigé à partir de 1830 par Jules Guérin lui-même, qui transforme l'ancienne Gazette de santé, et revendique l'application à la médecine d'un éclectisme philosophique. La recension des articles traitant de la question orthopédique et/ou gymnastique dans les colonnes de la Gazette médicale de Paris dépasse le cadre restreint d'un tel article, mais nous avons pu localiser plusieurs dizaines d'articles témoignant du questionnement orthopédique.

${ }^{41}$ A Rouen, Dijon, Lyon, ou Montpellier.

42 Ténotomie ou méthode chirurgicale pour le traitement des déviations. 
Genèse d'un champ scientifique : l'Orthopédie comme fabrique d'une gymnastique.

exercices d'une gymnastique prudente. L'Académie des Sciences focalise une partie des débats, en proposant en 1831 la mise au concours de travaux permettant de faire apprécier exactement la valeur de différentes méthodologies de redressement pour le traitement des difformités de la colonne vertébrale. Suite à cela, le débat peut-être le plus véhément ${ }^{43}$ opposera Guérin et un certain Hossard, et questionnera particulièrement les compétences orthopédiques de ce dernier, qui n’est pas médecin. Le fabricant de ceintures à levier Jules-Louis Hossard se vante de posséder une vraie légitimité au nom de la tutelle exercée par son père - médecin - au sein de son établissement orthopédique à Tours, mais cela ne suffit pas à convaincre les autorités médicales du temps, davantage bienveillantes pour les innovations proposées par les médecins-orthopédistes. Si l'Académie donne raison à Jules Guérin, il est finalement condamné - au pénal - pour diffamation, pour avoir traité M. Hossard de «charlatan » dans l'une de ses lettres expliquant - à raison - les supercheries mises en place par Hossard pour illustrer l'efficacité de sa méthode orthopédique ${ }^{44}$. Cela l'amène au commentaire suivant : «Le caractère appareillé de l'orthopédie a facilité l'entrée de non-médecins dans l'espace de pratique, ce qui a beaucoup décrédibilisé cette branche de la médecine, à cause d'échecs ou d'usages abusifs des lits à extension qui, même mécanisés, nécessitent une bonne connaissance du corps humain et de son fonctionnement. " ${ }^{45}$

Comme précédemment, nous voyons là toute l'intensité discursive qui marque la fondation d'une spécialité médicale, particulièrement autour de la compétence médicale (attestée par le diplôme de docteur), passeport quasiexclusif pour un art bien maîtrisé. La reconnaissance des pairs s'exprime par la validation d'une thérapeutique auprès de l'Académie, et atteste des luttes pour la captation d'un capital spécifique au champ médical et pour la fondation d'une spécialité aux pouvoirs thérapeutiques fondés. Sorcier parmi les sorciers, Hossard n’accède pas à la reconnaissance de l'institution ; alors que, par

${ }^{43}$ Le débat se déroule en 1835, au début du mois de septembre, dans les séances hebdomadaires de l'Académie royale de médecine. Lire tout particulièrement, l'« Avertissement» de l'ouvrage : Guerin, Jules. 1838. Mémoire sur les déviations simulées de la colonne vertébrale, et les moyens de les distinguer des déviations pathologiques, Paris, Bureau de la gazette médicale.

${ }^{44}$ Hossard réalise notamment de fausses moulures de dos déviés puis redressés par ses soins, une supercherie rapidement mise au jour par les regards experts des académiciens.

${ }^{45}$ GUERIN, Jules. 1835. «Travaux académiques - Orthopédie : Lettres sur quelques supercheries orthopédiques », Gazette médicale de Paris, tome 6, p. 623. 
Revue Sciences sociales et sport $n^{\circ} 2$ - Septembre 2009

ailleurs, parmi les prophètes les controverses s'engagent autour de la description des maladies et de leur expression musculaire, mais aussi sur la réponse à apporter à ces pathologies - chirurgicales ou non ${ }^{46}$. Mais encore les traitements de Hossard sont-ils trop exclusifs du mouvement, trop rigides d'une certaine manière, ils ne laissent pas de place à une mise en mouvement rationnelle et prudente des corps atteints de difformités. Touts chirurgiens ou mécaniciens qu'ils soient, les médecins introduisent systématiquement une part de gymnastique raisonnée dans leurs méthodologies de redressement. Jules Guérin sera encore amené à fréquenter les tribunaux dans les années 1840, après qu’il ait décidé de s'engager dans un procès contre les docteurs Malgaigne, Vidal de Cassis et Henroz, ces derniers ayant ouvertement critiqué sa ténotomie rachidienne ${ }^{47}$.

D'autres controverses existent, notamment autour de la fondation d'établissements orthopédiques et gymnastiques dans Paris, au cours de la première moitié du $\mathrm{XIX}^{\mathrm{e}}$ siècle. Celles-ci témoignent de l'existence d'un marché du redressement des corps et illustrent la concurrence qui oppose les différents directeurs, notamment en fonction de leur légitimité médicale exprimée dans la possession ou non du doctorat de médecine - et dans l'efficacité des méthodes développées. Madame Masson de la Malmaison unique femme à se lancer dans la direction d'un établissement orthopédicogymnastique - fait appel à différents médecins dans ses publications ${ }^{48}$, afin que ceux-ci viennent apporter une sorte de caution, à la fois morale et scientifique, à ses réalisations. Il va de soi qu'au sein de ses établissements Madame Masson de la Malmaison mène ses thérapeutiques en autonomie, et les membres de l'Académie de médecine, qui cautionnent son action, ne se déplacent que très rarement. Ce qui fait dire à d'autres directeurs d'établissements - dont Vincent Duval -, toute l'inquiétude qui est la leur quant à la direction des exercices

\footnotetext{
46 BouviER, Sauveur-Henri-Victor ; GuERIN, Jules. 1843. «Travaux académiques Académie de médecine - séance du 11 juillet : Déviations de l'épine-myotomie », Gazette médicale de paris, tome 14, p. 454-456

47 SACHAILE DE LA BARRE [pseudonyme de Claude LACHAISE]. 1845. Les médecins de Paris jugés par leurs œuvres ou statistique scientifique et morale des médecins de Paris, Paris, l'auteur, p. 348.

${ }^{48}$ Dont le baron Guillaume Dupuytren, comme nous l'avons vu précédemment, mais aussi d'autres membres de l'Académie de médecine (le docteur de Saint-Antoine, ou même une commission de l'Académie de médecine composée des docteurs-médecins : Bricheteau, Deleur et Thillaye). Voir, MASSON DE LA MALMAISON, Madame. 1840. Des difformités de la taille et des maladies qui les font naître, Paris, l’auteur.
} 
Genèse d'un champ scientifique : l'Orthopédie comme fabrique d'une gymnastique.

orthopédiques par Madame Masson de la Malmaison - même si cette dernière n'est pas explicitement nommée - :

« Il est à Paris une maîtresse de pension de jeunes demoiselles qui cherche à attirer des personnes contrefaites, en annonçant sa maison sous la direction d'un chirurgien connu par ses places, et, il faut le dire par son talent, hors toutefois, l'orthopédie, mais il faut en croire le bruit public, il ne serait pas désintéressé dans le succès de cet établissement, et la direction de ce chirurgien consisterait tout au plus dans une visite par semaine. ${ }^{49}$ Et l'expérience pédagogique de 20 années auprès de jeunes enfants ${ }^{50}$ ne pèse pas bien lourd, dans un marché où le capital spécifique - proprement médical -, et les savoirs pratiques des différents acteurs s'affirment de plus en plus comme structurant les relations de concurrence. Les exercices physiques, que Madame Masson de la Malmaison donne dans ses leçons, sont certes adaptés à son public et réfléchis en fonction de leur destination, mais ils ne suffisent pas à construire une vraie légitimité sur le marché.

Nos constatations empiriques rejoignent donc assez sensiblement les analyses de Patrice Pinell ${ }^{51}$ sur le champ médical dans son ensemble et sur le processus de spécialisation qu'il connaît depuis la fin du XVIII ${ }^{\mathrm{e}}$ siècle, ainsi que les propos de Pierre Bourdieu sur la genèse et la structuration du champ religieux ${ }^{52}$.

Nos premières analyses attestent de luttes autour d'un capital spécifique qui n'est pas exclusivement scientifique, mais également pratique, voire

49 DuVAL, Vincent ; JALADE-LAFOND, Guillaume. 1829. Etablissement orthopédique, Paris, l'auteur, p. 4.

${ }^{50}$ Masson de la Malmaison, Madame. 1831. Aperçu sur l'éducation physique des jeunes demoiselles, Paris, Plassan et Cie., p. 5.

${ }^{51}$ PINELL, Patrice. 2005. Art. cit.

${ }^{52}$ Pierre Bourdieu a confronté les écrits sur la religion de Marx, Weber et Durkheim pour forger son concept de « champ », lire particulièrement BouRdiEU, Pierre. 1971a. Art. cit.

A propos des similitudes avec le champ religieux, on soulignera tout particulièrement le processus de dépossession objective du capital spécifique par les plus légitimes dans le champ ouvert : «[...] la constitution d'un champ religieux est corrélative de la dépossession objective de ceux qui sont exclus et qui se trouvent constitués par là même en tant que laïcs [ou profanes, au double sens du terme - comme pour le colonel Amoros] dépossédés du capital religieux [comme travail symbolique accumulé - par les principaux contributeurs du processus de spécialisation de l’orthopédie] et reconnaissant la légitimité de cette dépossession du seul fait qu’ils la méconnaissent comme telle. ». BouRdiEU, Pierre. 1971a. Art. cit., p. 304-305. 
Revue Sciences sociales et sport $n^{\circ} 2$ - Septembre 2009

artistique, dans la mesure où la médecine est un art et où l'orthopédie participe d'un redressement des corps, qui doit aussi être pensé comme esthétique, et d'un intérêt commun aux différents acteurs, soit la construction et la validation de la méthodologie thérapeutique la plus performante pour les difformités du corps humain et particulièrement de la colonne vertébrale.

Des positionnements et des luttes que nous avons essayé de formaliser dans un espace à deux dimensions (figure 2, figure 3) qui distribue les médecins et les autres acteurs de la spécialisation orthopédique selon leur légitimité dans le champ médical et selon leur affinité avec les méthodes de redressement mécanique ${ }^{53}$.

Figure 2. Espace des prises de position orthopédico-gymnastique

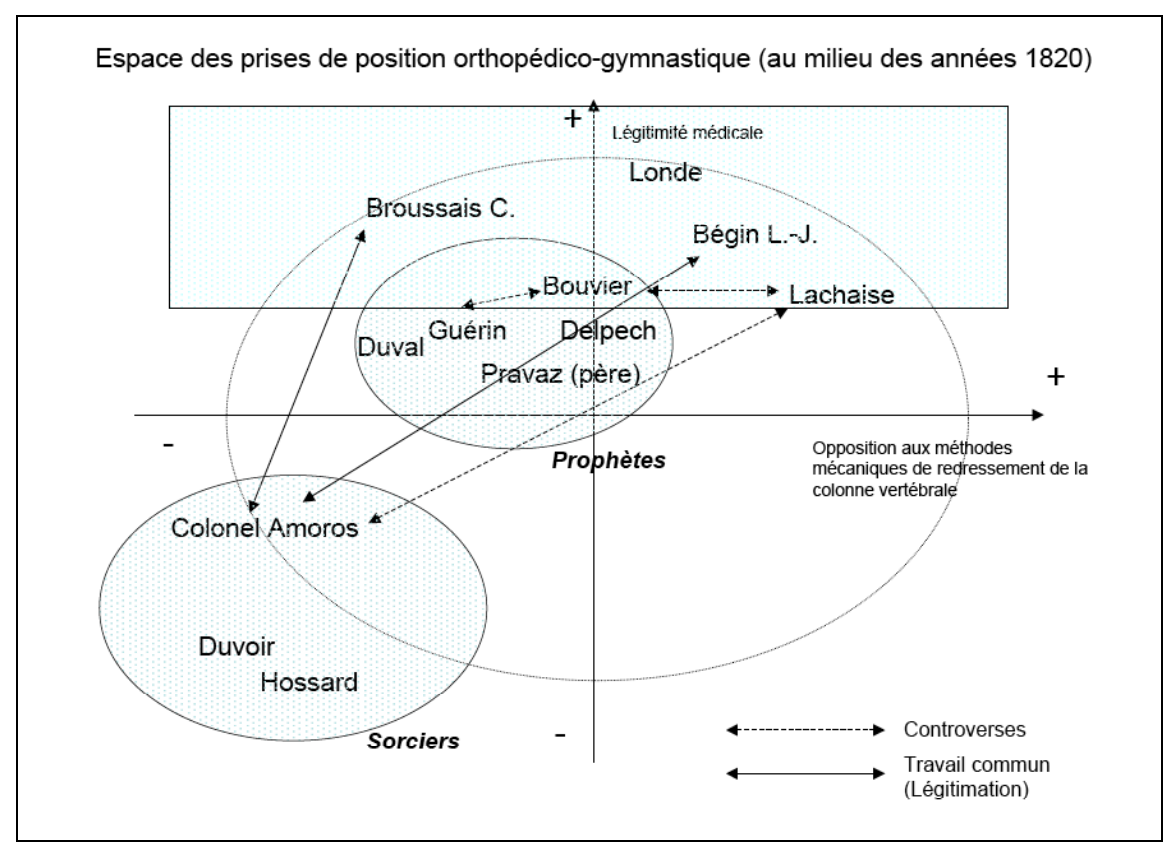

\footnotetext{
${ }^{53}$ Les schémas proposés dans cet article ne sont pas le résultat d'analyse statistique. Leur portée n'est qu'heuristique et vise à illustrer nos propos, en proposant une représentation graphique des controverses et des collaborations à deux moments de l'histoire du processus d'institutionnalisation de l'orthopédie.
} 
Genèse d'un champ scientifique : l'Orthopédie comme fabrique d'une gymnastique.

Il ne nous a pas semblé pertinent de formaliser l'espace selon un axe qui préciserait l'empathie pour les thérapeutiques gymnastiques, dans la mesure où un certain consensus semble exister, du moins dans les décennies qui nous concernent, et que ce consensus ne semble pas mis à mal par les innovations des uns et des autres. Les innovations contribuent davantage à diversifier les emplois des pratiques d'exercice corporel.

A partir de ces controverses et de ces collaborations, il semble difficile de raisonner strictement et exclusivement à partir de l'opposition «médecins contre praticiens ». Mais dans le cadre d'un champ en cours de structuration, il peut être intéressant de se pencher sur le rapport des différents acteurs à l'innovation orthopédique - étiologiques, séméiologiques, thérapeutiques, etc. et à l'innovation et la systématisation des pratiques d'exercice corporel.

\section{III - Sur l'innovation et la dynamique discursive. L'orthopédie et les exercices corporels}

L’orthopédie, établie par Nicolas Andry au milieu du XVIII ${ }^{\mathrm{e}}$ siècle, se développe notamment dans la seconde moitié du XVIII siècle, participant d'un mouvement biopolitique, d'une nouvelle gouvernementalité où la "population » s'impose "comme un ensemble de processus qu'il faut gérer dans ce qu'ils ont de naturel et à partir de ce qu'ils ont de naturel $»^{54}$. Les progrès s'expriment notamment à travers la conception d'un large éventail de choix thérapeutiques. Si les réflexions étiologiques et sémiologiques restent encore parcellaires au XVIII ${ }^{\mathrm{e}}$ siècle, Andry et ses continuateurs développent différentes méthodes de redressement, du lit mécanique aux mouvements réfléchis des corps. De nouvelles pratiques de santé émergent, et notamment des exercices corporels, ce n'est pas « encore véritablement "de la gymnastique", mais [...] un ensemble d'activités, qui ne sont pas pour autant des pratiques erratiques, avec des références scientifiques, avec des références "rationalisées" sans être nécessairement "savantes" " ${ }^{55}$. Toujours est-il que les principales innovations orthopédiques du XVIII ${ }^{\mathrm{e}}$ siècle demeurent encore ailleurs : le " lit mécanique » ou le " lit à extension » et le corset redresseur sont les techniques de redressement privilégiées. Ce qui ne va pas sans ambiguïté dans un espace

\footnotetext{
${ }^{54}$ FOUCAULT, Michel. 2004. Op. cit., p. 72.

${ }^{55}$ VigARELLO, Georges. 2004. « Le corps et ses représentations dans l'invention de la gymnastique », in POCIELlO, Christian (Dir.). Entre le social et le vital. L'éducation physique et sportive sous tension (XVIII ${ }^{e}-X X^{\mathrm{e}}$ siècle), Grenoble, PUG, p. 27.
} 
Revue Sciences sociales et sport $n^{\circ} 2$ - Septembre 2009

médical qui, par ailleurs, critique très fortement le port du corset et cherche à en bannir l'usage dans les pratiques pédagogiques. D'une part, nous avons une posture hygiénique (ou hygiéniste) qui prône une libération des corps dès la petite enfance ; et, d'autre part, nous avons le développement d'un souci thérapeutique - articulé à l'affermissement de la théorie localiste des pathologies $^{56}$ - qui doit contraindre le corps, lorsque le besoin s'en fait sentir.

Appareils à extension verticale ou horizontale, l'ensemble de l'Europe de la seconde moitié du XVIII ${ }^{\mathrm{e}}$ siècle voit différents acteurs de l'espace social médical forger différents moyens pour redresser mieux et plus durablement les affections orthopédiques, et tout particulièrement celles de la colonne vertébrale. Un certaine Heine développe un lit mécanique en Prusse, les docteurs Bampfield et Shaw en Angleterre proposent plus qu'une simple extension de la colonne, avec aussi quelques exercices propres à régulariser l'action musculaire. Mais c'est toujours empiriquement que se développent les innovations, avant le $\mathrm{XIX}^{\mathrm{e}}$ siècle, ainsi que pendant les premières décennies du $\mathrm{XIX}^{\mathrm{e}}$ siècle, et la production "savante » sur les affections orthopédiques reste très réduite. En 1819, dans l'article « orthopédie » du Dictionnaire des sciences médicales, François Fournier-Pescay et Louis-Jacques Bégin ne citent qu'un ouvrage en plus du traité d'Andry, à savoir celui de Desbordeaux : Nouvelle orthopédie, ou Précis sur les difformités que l'on peut prévenir ou corriger dans les enfants [publié en 1805]. Et encore, ils le citent pour dire qu'il « est écrit d'une manière trop vague [...] ; [et que] le cadre dans lequel il a voulu se renfermer est trop étroit pour que son livre soit aussi utile que l'importance de la matière pourrait le permettre ${ }^{57}$.

Ce sont les cinq prophètes qui vont transformer la discipline orthopédique et poser les jalons d'une éducation physique rationnelle et systématiquement adaptée à des besoins précis. Promoteurs d'un discours à la fois compréhensif et normatif, ils vont développer l'étiologie et la séméiologie des diverses affections orthopédiques, recherchant les causes et les symptômes, tout en essayant de développer un appareillage hygiénique et thérapeutique efficient. Delpech, Duval, Guérin, Pravaz et Bouvier sont ainsi des puissants vecteurs d'innovations ; ils forgent un moment orthopédique, qui procède d'acquis du XVIII ${ }^{\mathrm{e}}$ siècle, mais, au final, ils innovent davantage qu'ils n'empruntent au passé. A eux cinq, ils vont transformer l'intelligibilité que l'on peut avoir de diverses affections du squelette et du corps en général, puisqu’il

\footnotetext{
${ }^{56}$ PINELL, Patrice. 2005. Art. cit., p. 5-6.

${ }^{57}$ FourniER-PESCAY, François ; BEGIN, Louis-Jacques. 1819. Op. cit., p. 296-297.
} 
Genèse d'un champ scientifique : l'Orthopédie comme fabrique d'une gymnastique.

s'agit bien de «conserver l'intégrité des formes du corps [...] [et] éviter les causes capables d'altérer la conformation [...] [et] rechercher les influences les plus favorables à son développement régulier ${ }^{58}$. Ils vont publier divers articles dans les dictionnaires médicaux : « orthopédie » et " gymnastique », mais aussi " pied-bot », « rachis », ou encore « torticolis » et développer leurs recherches dans des établissements orthopédiques et gymnastiques.

Le premier, Jacques Mathieu Delpech, renforce le statut de la gymnastique comme support potentiel d'une guérison complète, mais la consultation de son ouvrage principal $^{59}$ montre aussi toute la confiance qu'il conserve dans les lits mécaniques et les appareils à extension et/ou à pression.

" [Il] est difficile de se faire une idée de l'enthousiasme qu'ont excitée en leur temps les lits orthopédiques pour le traitement des déviations de la taille. Il n'y avait pas, dit Jules Rochard, dans son histoire de la chirurgie française, [en] 1875, d'institution de jeunes filles, et même de jeunes gens qui crût pouvoir se passer de cet appareil. D'autre part, les brillants succès de la ténotomie [après 1835] excitèrent dans le monde savant une légitime attention, et c'est ainsi que, portée par la faveur publique, soutenue par l'opinion éclairée, l'orthopédie réussit non pas à forcer, mais à entrebâiller la porte de l'hôpital des Enfants. Un service extraordinaire orthopédique de douze lits fut créé et confié à Jules Guérin. [...] En un mot, il fonda avec [...] éclat l’Ecole orthopédique de l’hôpital des Enfants Malades. ${ }^{60}$

Pourtant, Jules Guérin est temporairement congédié en 1848, alors même qu'un rapport au gouvernement provisoire soulignait la qualité et la nouveauté de ses méthodes, procédés et appareils ${ }^{61}$. Son collègue à l’hôpital des Enfants Malades, Vincent Duval - ancien élève de François Broussais et

\footnotetext{
${ }^{58}$ BouviER, Sauveur-Henri-Victor. 1834. « Orthopédie », Dictionnaire de médecine et de chirurgie pratiques [Le Quinze Volumes ou Andral], Paris, Gabon, tome 12, p. 288.

${ }^{59}$ DELPECH, Jacques-Mathieu. 1828. L'orthomorphie par rapport à l'espèce humaine [...], Paris, Gabon. Le tome second, propose une partie « Des extensions et des pressions en général », parmi les thérapeutiques.

${ }^{60}$ SAINT-GERMAIN, L.-A. 1883. Chirurgie orthopédique thérapeutique des difformités congénitales ou acquises. Leçons cliniques professées à l'hôpital des Enfants malades, Paris, Baillière, p. 15-17.

${ }^{61}$ Blandin ; Dubois ; Jobert ; LOUIS ; RAYer ; Serres ; Orfila. 1848. Rapport adressé à Monsieur le délégué du gouvernement provisoire sur les traitements orthopédiques de M. le docteur Jules Guérin, à l'hôpital des Enfants, pendant les années 1843, 1844 et 1845, Paris, Bureau de la Gazette médicale, p. 201-202.
} 
Revue Sciences sociales et sport $n^{\circ} 2$ - Septembre 2009

spécialiste du pied-bot - développe également des méthodes chirurgicales, réalisant - en 1835 - pour la première fois en France la section sous-cutanée du tendon d'Achille. De fait, la trajectoire de Vincent Duval est principalement remarquable pour sa participation à l'institutionnalisation des diagnostics et des pratiques orthopédiques ; dès 1831, il dirige les traitements orthopédiques de l'hospice des Orphelins, et siège au bureau central d'admission. De son côté, Charles Gabriel Pravaz utilise les lits mécaniques, mais c'est surtout pour les améliorations qu'il va y apporter qu'il est reconnu. Sa principale innovation mérite d'être soulignée, car elle va bouleverser complètement l'usage des lits mécaniques, en introduisant une vraie réflexion en terme de mouvement, de sensibilité aux déplacements musculaires, de gymnastique rationnelle : « [...] La méthode de Pravaz [...] présente trois différences sur lesquelles repose toute sa supériorité :

- Les appareils de M. Pravaz sont construits de manière à permettre l'emploi simultané de la gymnastique et de l'extension de l'épine.

- La seconde différence consiste dans la construction même de ces appareils, c'est-à-dire dans la division qu'ils présentent transversalement, division qui permet de localiser l'extension.

- La troisième différence est philosophique, c'est-à-dire qu'elle repose sur une manière large et complète d'envisager les difformités de l'épine. Par une étude approfondie de ces maladies, M. Pravaz est parvenu à déterminer les diverses conditions et circonstances de leur formation et de leur développement ; il a tenu compte de toutes ces circonstances comme d'autant d'indications à considérer les unes par rapport aux autres, et sur lesquelles doivent reposer l'ensemble du traitement orthopédique. C'est ainsi que ce médecin ne se borne ni à l'emploi de ces appareils, ni à la gymnastique, parce que dans l'étude des déviations spinales il va chercher la cause de ces déviations au-delà de leur manifestation locale, en un mot, il embrasse toute la maladie depuis son origine jusque dans ses moindres symptômes, et approprie à chacun de ces éléments pathologiques les ressources que lui fournissent et la thérapeutique générale et les appareils orthopédiques et gymnastiques qu'il a inventés. " ${ }^{62}$

Charles Gabriel Pravaz concourt également à la diffusion des savoirs orthopédiques en province, par l'ouverture d'un établissement à Lyon. En outre, les noms de Duval et de Pravaz seront associés aux pratiques orthopédiques et

\footnotetext{
${ }^{62}$ Pravaz, Charles-Gabriel. 1833b. « Etablissement orthopédique et gymnastique - à
} Paris, rue de bellefond, n 32 », Gazette médicale de paris, tome 4, p. 755. 
Genèse d'un champ scientifique : l'Orthopédie comme fabrique d'une gymnastique.

gymnastiques dans la seconde moitié du XIX ${ }^{\mathrm{e}}$ siècle par les activités de leurs fils, respectivement Emile et Jean-Charles-Théodore. Tous deux continueront à s'engager pour l'orthopédie et l'éducation physique, perfectionnant même les innovations de leurs aînés, comme avec le bain à air comprimé et la sensibilité aux mouvements des corps pour Jean-Charles-Théodore Pravaz. Ce dernier déclare en 1873 : «Loin d'être bornée à l'application banale d'appareils, l'orthopédie, considérée au point de vue du traitement des difformités, invoque à son aide toutes les ressources de l'hygiène et de la médecine proprement dite, et, envisagée dans la plus haute expression, se trouve intimement liée à l'éducation physique. ${ }^{63}$

Le cinquième prophète Sauveur-Henri-Victor Bouvier est peut-être le plus complet dans la mise en oeuvre d'une compréhension critique des affections et dans la diversité des méthodologies thérapeutiques expérimentées : « Docteur à Paris en 1823, disciple [du chirurgien] Béclard, [Bouvier est] [...] connu comme habile anatomiste et se destinant à l'enseignement ; il va changer de voie alors que l'orthopédie, depuis longtemps négligée en France, devint tout à coup, pour quelques spéculateurs habiles, un assez bon moyen de fortune. " ${ }^{64}$

Bouvier va œuvrer dans toutes les directions : lits mécaniques, chirurgie, gymnastique, etc. Esprit de synthèse, il se démarque aussi pas des prises de position parfois tranchées, mais argumentées et non dogmatiques. Ainsi, dans un premier temps, il s'oppose également aux critiques du docteur Lachaise à propos des lits mécaniques - dans les années 1820 -, en le traitant de visionnaire, avant de le rejoindre et d'ajouter un gymnase à ses propres moyens de redressement. A la fin du XIX ${ }^{\mathrm{e}}$ siècle, le chirurgien de Saint-Germain, dit de lui : « [...] que l'orthopédie a gagné dans sa cohabitation avec un maître comme Bouvier. Il l'a fondée sur l'anatomie pathologique, sans laquelle il n'y a plus aujourd'hui d'enseignement ex professo possibles des sciences médicales, il l'a nourrie de son érudition, enrichie d'un riche bagage de toute sorte, embellie de son style correct, châtié, élégant. [...] Il en a fait une science. Elle ne peut plus, après lui, retomber dans l'empirisme pur. Tout au plus peut-elle, au contact du chirurgien, devenir plus alerte, plus expéditive, plus efficace, mais elle restera au fond ce que Bouvier l'a faite. Aussi c'est pour moi un devoir autant qu'un plaisir que d'insister avec détail sur la vie de cet homme remarquable. »"

\footnotetext{
${ }^{63}$ Pravaz, Jean-Charles-Théodore. 1873. De l'orthopédie, Lyon, Pitrat, p. 4.

${ }^{64}$ SACHAILE DE LA BARRE [pseudonyme de Claude LACHAISE]. 1845. Op. cit., p. 137138.

${ }^{65}$ SAINT-Germain, L.-A. 1883. Op. cit., p. 24-27
} 
Revue Sciences sociales et sport $n^{\circ} 2$ - Septembre 2009

Parmi les prophètes, Bouvier est aussi celui qui bénéficie le plus complètement de l'investissement de l'Académie de médecine dans les années 1830 et 1840, à travers des lectures critiques de ses travaux et expériences. Les Académies (de médecine et des sciences) contribuent notamment à valider ses innovations thérapeutiques et à légitimer ses réflexions, ainsi Victor Bouvier a «[...] eu la satisfaction de voir le succès couronner ses efforts [...] par le suffrage élevé de l'Académie Royale des Sciences qui, en 1837, lui a décerné un prix de 6000 francs pour ses travaux et ses découvertes en orthopédie $[\ldots] »^{66}$.

Les stratégies et trajectoires des acteurs du sous-espace de l'orthopédie s'articulent avec un processus d'institutionnalisation de savoirs et de pouvoirs sur les corps. Une lecture consécutive des deux représentations du champ orthopédique voit les prophètes suivre une trajectoire ascendante dans la légitimité médicale, et voit s'accroître la méfiance à l'encontre des méthodes strictement mécaniques. Et, nous soulignons encore que le processus est pluriel, à la fois vers une plus grande technicisation des techniques chirurgicales (Jules Guérin), et vers une plus grande souplesse dans l'usage (imaginé ou supposé) rationnel des exercices du corps (comme chez Charles Pravaz ou Victor Bouvier). Porteurs d'un discours de plus en plus systématique, les prophètes se muent en prêtres, et tendent à se distinguer davantage des sorciers ; un processus, illustré par l'accroissement de l'écart entre le groupe des prophètes et celui des sorciers, et par la progression des prophètes sur l'axe de la légitimité médicale.

L'institutionnalisation d'un champ médical sous la Restauration, puis sous la Monarchie de Juillet, implique le développement de relations de concurrence. Les effectifs de la Faculté de médecine de Paris explosent en quelques années $^{67}$, et l'orthopédie - spécialité en voie d'institutionnalisation n'échappe pas à ce phénomène, dont l'une des conséquences, et peut-être la première, est l'innovation interne à la spécialité, comme voie de distinction individuelle et collective.

${ }^{66}$ Bouvier, Sauveur-Henri-Victor. 1840. Institut orthopédique de Chaillot, pour le traitement des difformités de la taille et des membres, dirigé par M. le docteur Bouvier, Paris, Pillet. Le docteur Jules Guérin a obtenu le premier prix de 10.000 francs, mais les académiciens demeurent circonspects devant les conséquences encore peu étudiées des sections musculaires et tendineuses de Guérin.

${ }^{67}$ LEONARD, Jacques. 1966. " Les études médicales en France entre 1815 et 1848 », Revue d'histoire moderne et contemporaine, tome 13, n 1 , p. 87-94. 
Genèse d'un champ scientifique : l'Orthopédie comme fabrique d'une gymnastique.

Figure 3. Espace des prises de position orthopédico-gymnastique (fin des années 1830).

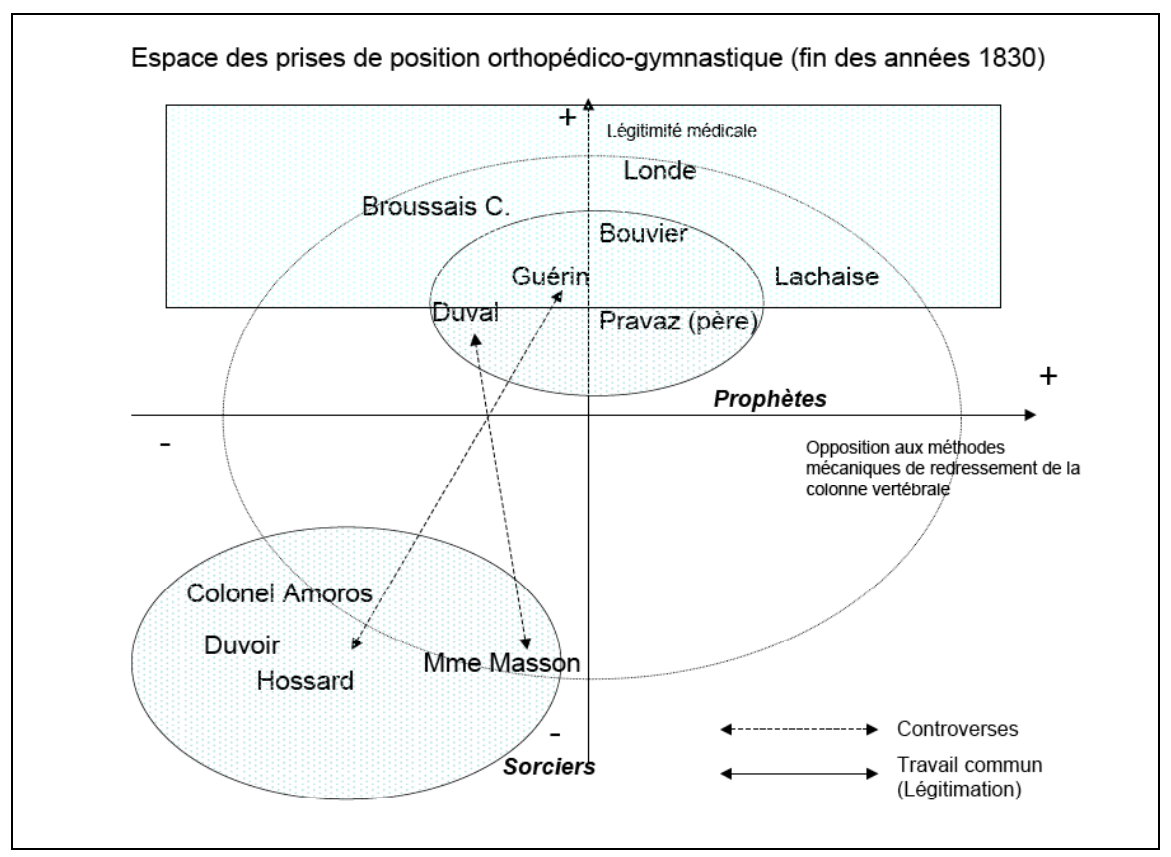

Le Gymnastique ne peut être considérée que comme une semi-novation de l'orthopédie, dans la mesure où d'autres espaces sociaux - en dehors même de la médecine - contribuent aussi à son développement. Ce qui semble se produire au sein de la spécialité orthopédique c'est plutôt un renforcement du modus vivendi autour de son utilisation comme moyen d'appoint et de renforcement des résultats thérapeutiques obtenus par ailleurs, et une diversification des modalités de pratiques selon les états pathologiques, et plus largement selon la constitution, selon l'âge, selon le sexe, dans un processus de systématisation de la mise en mouvement des corps. L'opposition aux méthodes strictement mécaniques de redressement s'accompagne d'une plus grande attention portée sur l'efficience hygiénique et thérapeutique des exercices du corps et sur la connaissance physiologique que les médecins ont du corps humain. Ainsi, l'intelligibilité que les médecins ont du flux sanguin régulier les menstrues - interfère par exemple avec la mise en place de thérapeutiques 
Revue Sciences sociales et sport $n^{\circ} 2$ - Septembre 2009

gymnastiques. Charles Gabriel Pravaz rappelle « [...] que, dans le cas des jeunes filles délicates, l'usage de la gymnastique et des lits à extension [mais surtout de la gymnastique] entraîne un arrêt provisoire du flux menstruel [...] ${ }^{68}$, or ce défaut d'écoulement n'est pas interprété comme potentiellement pathologique, mais bien plus comme contribuant au renforcement du corps par un apport nutritif accru. C'est alors l'effet secondaire de l'exercice physique qui est profitable aux corps féminins. Le «meilleur moyen de détruire les effets fâcheux que produisent souvent les excès intellectuels et moraux, c'est de faire faire au malade un exercice convenable. Combien d'hystériques, de mélancoliques, d'érotomanes, etc., n'ont-ils pas dû leur guérison à un genre de vie très actif qu'on les obligeait à suivre [...] »" ${ }^{69}$, précise encore le docteur Pravaz dans l'article « Gymnastique » qu'il rédige pour un dictionnaire médical. Il contribue, là encore, à accentuer l’intrication du médical et du pédagogique.

\section{Conclusion}

Dans les années 1820-1840, l’orthopédie participe à l'établissement d'une gymnastique en forme de grammaire des corps en mouvement, que l'institution scolaire généralisera dans la seconde moitié du $\mathrm{XIX}^{\mathrm{e}}$ siècle. Substrat d'un bon et prompt rétablissement, la gymnastique est pleinement engagée en première ligne sur le marché du redressement des corps. Elément d'union entre les acteurs du processus orthopédique, la gymnastique n'en est pas moins diversement employée. Hygiénique, elle doit être diffusée pour empêcher un plus grand développement des difformités dans l'ensemble du corps social ; et thérapeutique, elle peut, d'une part, jouer un rôle d'accompagnement des redressements ou, d'autre part, concourir à la pérennité des réussites d'opérations chirurgicales ou mécaniques. Si c'est la diversité des méthodes thérapeutiques qui donne du relief au processus de spécialisation de l'orthopédie, à l'intérieur du champ médical, la construction d'une mise en mouvement rationnelle des corps en parallèle de la recherche d'une thérapeutique convenable aux pathologies de la colonne vertébrale, contribue à la durable diffusion des normes par le travail d'incorporation explicite qui est mis en place.

\footnotetext{
${ }^{68}$ PrAVAz, Charles-Gabriel. 1833a. « Thérapeutique - De la gymnastique appliquée au traitement de quelques maladies constitutionnelles », Gazette médicale de paris, tome 4, p. 191.

69 Pravaz, Charles-Gabriel. 1836. «Gymnastique », Dictionnaire de médecine ou répertoire général des sciences médicales considérées sous le rapport théorique et pratique, Paris, Béchet Jeune, tome 14, p. 453-454.
} 
Genèse d'un champ scientifique : l'Orthopédie comme fabrique d'une gymnastique.

Au grand jamais, il ne faut voir dans cette esquisse de gymnastique un instrument de "libération corporelle ", anachronisme pour le XIX ${ }^{\mathrm{e}}$ siècle ; ce sont plutôt des discours en forme de (ré)assignation corporelle, à l'intérieur de canons esthétiques, moraux et sociaux créés par la bourgeoisie et pour la bourgeoisie.

L'essor d'un marché du redressement des corps, qui s'exprime notamment à travers le nombre de fondation de maisons de santé orthopédique et gymnastique entre 1820 et 1835 , se mue en fabrique d'exercices corporels à travers l'action hygiénique et thérapeutique et les discours des orthopédistes. Prophètes, sorciers ou prêtres, ils contribuent finalement ensemble à alimenter l'institutionnalisation de l'orthopédie et la systématisation des pratiques d'exercice corporel. Et, ce faisant, ils évoluent eux aussi à l'intérieur du champ médical, ainsi les prophètes, qui développent un discours de plus en plus systématique et scientifique, vont accéder à la reconnaissance institutionnelle et parvenir à un statut de prêtre. De fait, à la fin de la décennie 1840, l'orthopédie semble être devenue un sous-champ médical légitime doté de ses savoirs et de ses lieux de pouvoir propres.

Très centrés sur un redressement technique des corps humains, les discours orthopédiques posent néanmoins des jalons d'un questionnement physiologique sur la dégénérescence de la race, davantage exacerbé au cours des décennies 1860 et 1870. L'orthopédie pose également la question de l'éducation des $\operatorname{corps}^{70}$ et, au-delà, des liens qui devraient exister entre médecine et pédagogie. Déjà réel avant la Révolution, le procès en intrication de la médecine et de la pédagogie, se réactualise ainsi sur un marché du redressement des corps, comme au sein du débat autour la phrénologie. Ainsi, les discours médicaux ou scientifiques autour des thèses de Franz Joseph Gall et de sa phrénologie sous-tendent ce procès, et il n'est pas surprenant de retrouver des acteurs du procès orthopédique au cœur de ce débat. Casimir Broussais, professeur de physiologie attaché au Gymnase du colonel Amoros, est également le premier secrétaire général de la société phrénologique dès 1831 ; alors que Sauveur-Henri-Victor Bouvier ${ }^{71}$ se pose davantage en opposant à la doctrine de Gall, comme d'ailleurs une majorité de ses confrères dès le début de la décennie 1830 .

\footnotetext{
${ }^{70}$ Fournier-PesCAY, François ; Begin, Louis-Jacques. 1819. Op. cit., p. 299-301.

${ }^{71}$ BOUVIER, Sauveur-Henri-Victor. 1839. Mémoire sur la forme générale du crâne dans ses rapports avec le développement de l'intelligence, Paris, Baillière.
} 
Revue Sciences sociales et sport $n^{\circ} 2$ - Septembre 2009

L'historicité des grandes doctrines scientifiques et médicales (phrénologie, vitalisme, positivisme, etc.), et leurs liens aux structures socioscientifiques et à l'éducation des corps sont évidemment riches, et invitent à l'approfondissement des analyses.

* Quin, Grégory, (doctorant) - Institut des Sciences du Sport et de l'Education Physique (ISSEP). Université de Lausanne - (gregory.quin@unil.ch). 